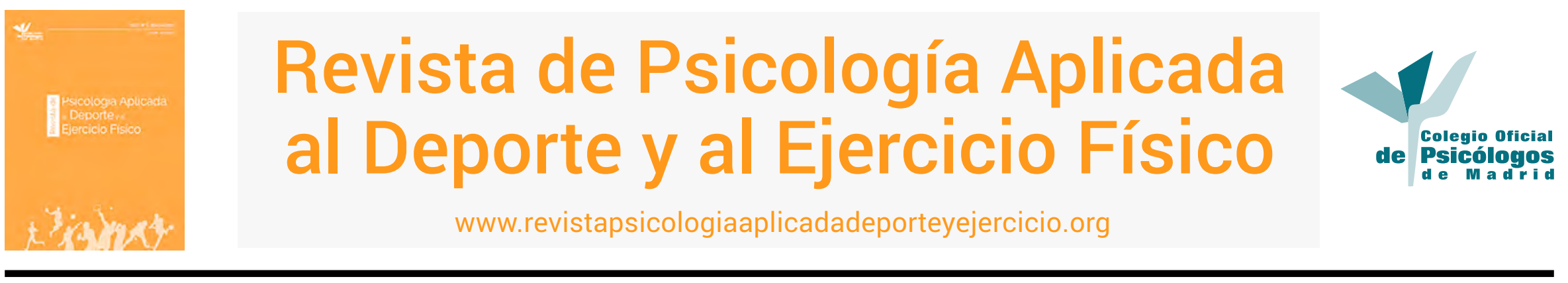

\title{
Diseño y validez de contenido de una entrevista para la evaluación psicológica de porteros de fútbol
}

\author{
M. Rocío Bohórquez Gómez-Millán', Irene Checa Esquiva² \\ ${ }^{1}$ Universidad de Sevilla, España, ${ }^{2}$ Universidad de Valencia, España
}

RESUMEN: Para la evaluación de las competencias psicológicas de los deportistas se han empleado diferentes instrumentos como los cuestionarios estandarizados, las técnicas de autoinforme construidas ad hoc o las anamnesis; aunque son estas últimas las que incluyen el mayor rango de conductas que definen el rendimiento deportivo y aquellas respuestas o estados que influyen en el mismo. Por ello, el objetivo de este trabajo fue crear un instrumento de evaluación con formato de entrevista semi-estructurada que recogiera todas aquellas necesidades de trabajo psicológico que pudieran tener los porteros de fútbol como jugadores específicos. La entrevista semi-estructurada se ha sometido a juicio de 4 expertos para su adecuación, así como el análisis de validez de los productos de la misma, a través de su aplicación a 4 porteros profesionales o semi-profesionales de fútbol, obteniendo niveles adecuados en ambos procedimientos. La naturaleza idiográfica de la entrevista creada permite obtener una visión específica y completa de la situación y contexto del portero de fútbol. Esta herramienta permitirá el diseño de programas de intervención orientados a una mejoría tanto cualitativa como cuantitativa de éstos deportistas concretos.

PALABRAS CLAVES: Entrevista, evaluación, factores psicológicos, porteros, fútbol.

\section{Design and content validity of an interview for the psychological evaluation of soccer goalkeepers}

ABSTRACT: Different instruments have been used to assess the psychological skills of athletes, including standardized questionnaires, ad hoc constructed self-report techniques, or anamneses. However, the latter include the greater range of behaviors that define sports performance and those responses or states that influence it. Therefore, the aim of this paper was to create a semi-structured interview as an assessment instrument that would cover all those psychological work skills that soccer goalkeepers might have as specific players. Four experts have judged the suitability of the semi-structured interview and analyzed the validity of its results by applying it to four professional or semi-professional soccer goalkeepers, obtaining adequate levels in both procedures. The idiographic nature of the interview created allows for a specific and complete view of the situation and context of soccer goalkeepers. This tool will allow for designing intervention programs aimed at improving both qualitative and quantitative competences of these specific athletes.

KEYWORDS: Interview, assessment, psychological factors, goalkeepers, soccer.

\section{Desenho e Validez do conteúdo de uma entrevista para a avaliação psicológica dos guarda-redes no futebol}

RESUMO: Para avaliar as capacidades psicológicas dos desportistas foram utilizados diferentes instrumentos como: questionários estandardizados; técnicas de auto-relatório construídas ad hoc e as anamnésias, apesar das últimas serem as que incluem o maior número de comportamentos que definem o rendimento esportivo e aquelas respostas ou estados que o influem nele. Por isso, o objetivo deste trabalho foi criar um instrumento de avaliação num formato de entrevista semi-estruturada que recolhesse todas as necessidades de trabalho psicológico que pudessem ter os goleiros de futebol como jogadores específicos. A entrevista semi-estruturada foi sometida ao parecer de 4

\footnotetext{
Este trabajo ha sido posible gracias al Proyecto "Asesoramiento psicológico para la mejora del rendimiento de los profesionales que prestan sus servicios en el Sevilla FC" con Referencia 2957/0809 financiado por el Sevilla FC SAD.

M. Rocío Bohorquez es psicóloga y pertenece al Departamento de Psicología Social en la Facultad de Ciencias de la Educación en la Universidad de Sevilla Irene Checa es psicóloga y pertenece al Departamento de Metodología y Ciencias del Comportamiento en la Universidad de Valencia.

La correspondencia sobre este artículo debe enviarse a la primera autora al Departamento de Psicología Social, Facultad de Psicología, Universidad de Sevilla. C/Camilo José Cela S/N, 41008, Sevilla.
}

(cc) EY-Nc-No Este es un artículo Open Access bajo la licencia 
dos peritos para a sua adequação, bem como à análise de validade dos produtos dela, através da sua aplicação a quatro goleiros de futebol profissionais ou semi-profissionais, e forma obtidos níveis adequados em ambos os procedimentos. A natureza ideográfica da entrevista realizada permite obter uma visão específica e completa da situação e do contexto do goleiro de futebol. Esta ferramenta irá permitir a construção de programas de intervenção orientados a uma melhoría tanto qualitativa como quantitativa destes desportistas concretos.

PALAVRAS-CHAVE: Entrevista, avaliação, factores psicológicos, goleiros, futebol.

Artículo recibido: 26/04/2017 | Artículo aceptado: 06/06/2017

En las últimas décadas, la investigación y el desarrollo de la evaluación en Psicología del Deporte ha avanzado de forma sustancial (Eklund, 2014), sin embargo, en la mayor parte de los casos, esta evaluación es realizada a través de cuestionarios (Tkachuck, Leslie-Toogood y Martin, 2003). Meredith, Dicks, Noel y Whagstaff (2017) Ilevaron a cabo una revisión de investigaciones publicadas en Psicología del Deporte en la que encontraron que del total de éstas desde el año 1973, sólo en el $14.7 \%$ usaron entrevistas como método de evaluación; este mismo estudio señaló que en los últimos años el interés por otros sistemas de evaluación profesional y por la investigación cualitativa había crecido considerablemente, como refrendan otros autores (Anguera, Camerino, Castañer y Sánchez-Algarra, 2014). Este aumento del interés suscita el desarrollo de instrumentos cualitativos de evaluación como las entrevistas adaptadas a los diferentes deportes (Cantón, 2016; Dosil, 2004; González, 2010; Tkachuck et al., 2003), que responden a la actual súper especialización del deporte, en búsqueda de resultados competitivos (Torregrosa, Sánchez y Cruz, 2004).

Para la detección de las necesidades de los deportistas se han empleado diferentes instrumentos como los cuestionarios estandarizados, las técnicas de autoinforme construidas ad hoc o las anamnesis (Meredith et al., 2017). Sea cual fuere el sistema de evaluación empleado para la detección de necesidades, éste debe incluir aquellas conductas que definen el rendimiento deportivo y aquellas respuestas o estados que influyen en el mismo (Garcés de los Fayos, 2006; Martin, 2008); de éstos factores, se tendrán en cuenta tanto los indicadores cognitivos como los fisiológicos, conductuales y emocionales (Meredith et al., 2017). La entrevista semi-estructurada como instrumento básico de evaluación en Psicología (general y del deporte) será idiográfica por naturaleza, enfatizando la visión específica e individual de la situación y el contexto de la persona a la que se evalúa, que permitirá el diseño de programas de intervención orientados a una mejoría tanto cualitativa como cuantitativa del deportista (Anguera, 2002; Tkachuck et al., 2003).

Puesto que cualquier programa de entrenamiento de habilidades psicológicas para deportistas se inicia con una fase de evaluación inicial o detección de necesidades (Jiménez y Garcés de los Fayos, 1997; Williams, 1991), contar con instrumentos adecuados de evaluación que aporten una visión global de la realidad del deportista resulta de enorme importancia. Puesto que estas necesidades serán diferentes para cada deportista al depender de sus características e historia deportiva personales, su modalidad deportiva, factores situaciones y factores de interrelación entre otros (Capdevila y Niñerola, 2006; García-Más, 2002), la entrevista semi-estructurada se postula como una herramienta clave en el proceso. Esta entrevista debe, además, ahondar en la importancia relativa de los aspectos mentales de cada disciplina deportiva (Sánchez y Torregrosa, 2005).

Las especificaciones del puesto del portero de fútbol incluyen aquellas acciones orientadas a evitar goles, ya sea con cooperación del resto de sus compañeros de equipo o en solitario (como en el caso de los penaltis), y a crear nuevas opciones de juego para su equipo (Bohórquez, 2016). La investigación que los psicólogos han llevado a cabo sobre este puesto específico se han centrado básicamente en las habilidades que se requerían para enfrentar con éxito la tarea de parar penaltis: focalizar la atención en los pre-índices del tirador como estrategia para anticipar la dirección del balón ( $\mathrm{Na}$ via, Ruiz, Graupera, y Van der Kamp, 2011; Ryu, Kim, Abernethy, y Mann, 2013); afrontar la decepción posterior al fracaso (Lidor, Ziv, y Gershon, 2012); tomar decisiones (Bar-Eli, Azar, Ritov, Keidar-Levin, y Schein, 2007); y manejar del rendimiento del rival mediante el uso -principalmente- del lenguaje no verbal (Furley, Dicks, y Memmert, 2012; Manley et al., 2008).

Por lo expuesto anteriormente, el objetivo de este trabajo es crear un instrumento de evaluación con formato de entrevista semi-estructurada que recoja todas aquellas necesidades de trabajo psicológico que pudieran tener los porteros de fútbol como jugadores específicos.

\section{Método}

\section{Participantes}

La muestra de participantes en este estudio ha sido de 4 porteros de fútbol de entre 19 y 25 años de edad $(M=21.62$, $D T=2.75)$. Los criterios de inclusión para formar parte de la muestra fueron: 1) ser portero de fútbol 11;2) pertenecer a un equipo profesional o semi-profesional de fútbol; 3 ) 
aceptar formar parte de la investigación y firmar el consentimiento informado. Estos porteros entrenaban 4 ó 5 días a la semana con una duración de los entrenamientos aproximada de 120 minutos. Tres de ellos tenían una situación habitual de titularidad mientras que el restante solía ser suplente. Aquellos datos sociodemográficos de la entrevista que podrían identificar a los participantes han sido omitidos en esta investigación.

\section{Instrumentos}

El instrumento de evaluación utilizado ha sido una entrevista semi-estructurada, de cumplimentación cara a cara, no dirigida, de estudio e individual (Anguera, 2002) creada adhoc para este trabajo. Partiendo de una anamnesis genérica de Psicología del Deporte, se contemplaron y adaptaron al portero los siguientes bloques:

- Bloque de datos personales y de contacto.

- Bloque de datos sociodeportivos: club, clubes anteriores, años de práctica, nivel competitivo, competiciones en las que el equipo participa, años en esa competición, número y duración de los entrenamientos a la semana, situación habitual en las convocatorias, lesiones actuales o recidivantes.

- Motivo de consulta y/o información de derivación.

- Motivación: causas del inicio y mantenimiento en la actividad deportiva, factores influyentes en los procesos motivacionales y manejo de las variaciones.

- Autoconcepto y autoestima: fortalezas y debilidades como portero.

- Transferencia de las habilidades deportivas y psicológicas del entrenamiento a la competición: estado del aprendizaje y control motor de las acciones específicas

- Atribuciones causales: atribuciones ante éxitos y fracasos, consecuencias conductuales cognitivas y afectivas, errores de atribución. Identificación de componentes de flow asociados a la mejor competición.

- Arousal: identificación de la zona óptima de funcionamiento individual, aspectos que sacan el portero de la zona, estrategias de recuperación del estado de óptimo funcionamiento.

- Toma de decisiones: procedimiento, claves, influencia de otros, influencia de las emociones.

- Ansiedad precompetitiva: presencia/ausencia, sintomatología, impacto en los componentes de entrenamiento invisible, impacto en la precompetición, impacto en la competición, impacto en la postcompetición y estrategias de control.

- Manejo de las situaciones competitivas: distracciones, estrategias de reenfoque, gestión del error, gestión del éxito, nivel de confianza percibido
- Cuestiones relacionadas con agentes deportivos influyentes: compañeros (incluyendo otro/s porteros), entrenador de porteros, primer entrenador, preparador físico, fisioterapeuta, otros técnicos, familia y otros pertenecientes a la red de apoyo social.

- Percepción de necesidades psicológicas a entrenar.

- Observaciones del psicólogo tras la evaluación inicial.

\section{Procedimiento}

El procedimiento comenzó con la revisión de la literatura previa sobre las necesidades psicológicas del portero de fútbol y los contenidos de las entrevistas adaptadas a Psicología del Deporte (Dosil, 2004). A partir de ahí, se diseñaron las categorías relevantes, paso imprescindible, según Anguera et al., (2014), en cualquier diseño cualitativo. Partiendo de esas categorías, se diseñó la entrevista estructurada piloto, que es presentada en este trabajo. El resultado final se sometió a juicio de expertos, tomándose como expertos dos psicólogos con experiencia aplicada en el fútbol y dos entrenadores de porteros de dilatada trayectoria. Estos expertos asignaron a cada una de las preguntas de la entrevista dos puntuaciones. En la primera de las valoraciones los expertos contestaron a la cuestión ¿En qué medida crees que esta pregunta es necesaria en una entrevista psicológica para porteros? con una escala entre 1 (Nada pertinente) y 4 (Totalmente pertinente). Por otra parte, los expertos respondieron a la cuestión ¿En qué medida crees que esta pregunta mide lo que pretende evaluar esta área?, con una escala igual a la anterior. En una segunda fase, la entrevista fue administrada a los cuatro participantes. Finalmente, las entrevistas fueron analizadas independientemente por dos evaluadoras con el fin de determinar la validez de las mismas.

El procedimiento de recogida de datos ha sido llevado a cabo a través de entrevistas concertadas con los porteros fuera de su horario y lugar de entrenamiento habitual, en el despacho de un psicólogo especializado en deporte. Cada entrevista fue registrada mediante un sistema de grabación de sonido. Se han recogido las pertinentes autorizaciones, asegurando la confidencialidad de todos los datos recogidos.

\section{Análisis de datos}

En primer lugar se llevó a cabo el análisis de los resultados de las puntuaciones asignadas por los expertos. Con los datos, se creó una matriz de concordancia y se calcularon los índices Kappa de Cohen de fiabilidad interjueces, de manera que se obtuvieran datos fiables acerca de la coherencia de las decisiones entre los revisores. Con esos datos, se di- 
señó la versión piloto de la entrevista que fue administrada.

Una vez llevadas a cabo las entrevistas, se revisaron las transcripciones y se procedió al análisis cualitativo. Las dos evaluadoras analizaron las entrevistas por separado y asignaron un valor a cada área utilizando una taxonomía con tres categorías: objetivo prioritario de trabajo psicológico, objetivo secundario de trabajo psicológico y área funcional. De esta forma, se pudo comprobar la utilidad aplicada de la entrevista en cada una de las áreas.

Tras los resultados obtenidos en ambas fases, se modificaron algunas de las preguntas y la entrevista final es presentada en el Anexo.

\section{Diseño de investigación}

Siguiendo a Ato, López y Benavente (2013) el diseño de investigación puede ser englobado dentro de la investigación instrumental, ya que este trabajo presenta las primeras evidencias cualitativas de un instrumento de evaluación. Utiliza metodología cualitativa a través del análisis de discurso utilizando la entrevista como método de recogida de datos.

\section{Resultados}

\section{Acuerdo interjueces}

El cálculo del kappa de Cohen presenta un acuerdo entre jueces de $\mathrm{k}=.75$ en la cuestión de adecuación de la necesidad de las preguntas en una entrevista a porteros de fútbol y un acuerdo $\mathrm{k}=.67$ en la adecuación de la preguntas al área asignada. Ambos valores son considerados como buenos (Cohen, 1960; Landis y Koch, 1977).

\section{Validez}

Para analizar la validez de la entrevista propuesta como herramienta de evaluación se llevaron a cabo análisis de la misma por parte de dos psicólogas especializadas en deporte, que identificaron objetivos prioritario, secundario y áreas funcionales para cada uno de los porteros de la muestra. Los resultados (Tabla 1) indicaron acuerdo entre los psicólogos evaluadores en un $69.44 \%$ de las observaciones realizadas, acuerdo que puede considerarse adecuado. En las situaciones de desacuerdo, los criterios seleccionados por los evaluadores fueron contiguos en todas las ocasiones (por ejemplo, si un evaluador calificaba el área como funcional el otro la calificaba como objetivo secundario, pero nunca prioritario).

Existen diferencias en la evaluación de los evaluadores en las áreas de Atribuciones Causales para el Portero 1, Transferencia de Habilidades para el Portero 2, Arousal para el Portero 2, Toma de Decisiones para los Porteros 2 y 4, Manejo de la competición para los Porteros 2 y 4, Autoconcepto para el Portero 3 y Ansiedad Precompetitiva para el Portero 4.

\section{Discusión}

En el proceso de evaluación de un deportista deben utilizarse diferentes métodos que aumenten la fiabilidad de la

Tabla 1. Identificación de áreas de trabajo de cada portero

\begin{tabular}{|c|c|c|c|c|c|c|c|c|}
\hline & \multicolumn{2}{|c|}{ PORTERO 1} & \multicolumn{2}{|c|}{ PORTERO 2} & \multicolumn{2}{|c|}{ PORTERO 3} & \multicolumn{2}{|c|}{ PORTERO 4} \\
\hline & E1 & E2 & E1 & E2 & E2 & E2 & E1 & E2 \\
\hline Motivación & $\mathrm{OP}$ & $\mathrm{OP}$ & $F$ & $F$ & $\mathrm{~F}$ & $\mathrm{~F}$ & OS & OS \\
\hline Autoconcepto y autoestima & $\mathrm{F}$ & $\mathrm{F}$ & $\mathrm{F}$ & $F$ & OS & $\mathrm{F}$ & OS & OS \\
\hline Transferencia de habilidades & $\mathrm{F}$ & $\mathrm{F}$ & $\mathrm{F}$ & OS & $\mathrm{F}$ & $\mathrm{F}$ & $\mathrm{F}$ & $\mathrm{F}$ \\
\hline Atribuciones causales & OS & OP & OS & OS & $\mathrm{F}$ & $F$ & OS & OS \\
\hline Arousal & $\mathrm{F}$ & $F$ & OS & $\mathrm{OP}$ & $\mathrm{OP}$ & $\mathrm{OP}$ & $\mathrm{OP}$ & $\mathrm{OP}$ \\
\hline Toma de decisiones & $\mathrm{F}$ & $F$ & $F$ & OS & $\mathrm{F}$ & $\mathrm{F}$ & $F$ & OS \\
\hline Ansiedad precompetitiva & $\mathrm{F}$ & $F$ & $\mathrm{~F}$ & $F$ & $\mathrm{~F}$ & $\mathrm{~F}$ & OS & $\mathrm{F}$ \\
\hline Manejo de la competición & $\mathrm{OP}$ & $\mathrm{OP}$ & $\mathrm{OP}$ & OS & $\mathrm{OP}$ & $\mathrm{OP}$ & $\mathrm{OP}$ & OS \\
\hline Interacción con agentes deportivos & $\mathrm{F}$ & $\mathrm{F}$ & OS & OS & $\mathrm{F}$ & $\mathrm{F}$ & $\mathrm{F}$ & $\mathrm{F}$ \\
\hline
\end{tabular}

Nota: $\mathbf{E} 1=$ evaluador $1 \quad$ E2 = evaluador $2 . \quad$ OP = objetivo prioritario $\quad$ OS = objetivo secundario $\quad \mathbf{F}=$ funcional. 
información obtenida (González, 2010). La entrevista es el instrumento de referencia para los profesionales de la Psicología, ya que recoge los datos más significativos y de más difícil acceso a través de otros métodos como cuestionarios o sistemas de observación (Perpiñá, 2014). En este trabajo se presenta el proceso de creación de una entrevista semi-estructurada dirigida a evaluar el desempeño de los porteros de fútbol en el plano psicológico. Para ello, se siguió un proceso que contiene las siguientes fases: a) revisión de la bibliografía existente; b) creación de las preguntas de la entrevista; c) juicio de expertos; d) administración piloto a cuatro porteros de fútbol; e) análisis de dos evaluadoras de forma independiente; y f) reformulación y ampliación de algunas partes de la entrevista tras analizar las diferentes decisiones que las evaluadores tomaron tras sus análisis.

Tras la creación de la entrevista y su valoración por parte de los expertos, éstos arrojaron índices de acuerdo buenos para la adecuación de las preguntas a las habilidades y necesidades de un portero de fútbol así como de las preguntas al área que habían sido adjudicadas. No se sugirieron modificaciones sustanciales a la entrevista por parte de los cuatro expertos, tan sólo se señaló alguna errata.

Una vez definida y estructurada la entrevista piloto, se entrevistó a los porteros participantes en el estudio. Las dos evaluadoras, tras llevar a cabo el análisis de forma independiente, ordenaron las áreas en función de la prioridad que interpretaban de sus respuestas. En algunas de las áreas las evaluadoras presentaron falta de acuerdo, aunque en la mayor parte de las cuestiones (casi el 70\%) existía consenso en cuanto a la prioridad de intervención; estos resultados son coherentes con los arrojados en otras validaciones de entrevistas fuera del ámbito deportivo (Angold y Costello, 2000; Ezpeleta, de la Osa, Doménech, Navarro y Losilla, 1997). Así, esta primera versión de la entrevista presenta validez de contenido, tanto a través del juicio de expertos previo como tras su administración a la muestra piloto de porteros de fútbol.

El análisis de los resultados obtenidos evidenció la necesidad de reformular algunas de las cuestiones planteadas. Concretamente, en el apartado de autoconcepto y autoestima, cuando los participantes eran cuestionados acerca de sus puntos fuertes y débiles como porteros, todos ellos se remitieron en primera instancia a competencias técnicas concretas como "el juego aéreo", "los blocajes bajos cercanos a mi posición" o "la lectura de los penaltis". En este aspecto, además, la elicitación de puntos débiles siempre resultó más productiva que la de puntos fuertes. Por este motivo, durante la entrevista fue necesario repreguntar para incluir aspectos tácticos, estratégicos, físicos y psicológicos e incluso en este último punto recurrir a ejemplos. En consecuencia, las investigadoras decidieron realizar modificaciones en la en- trevista semi-estructurada que se propone para facilitar la producción de información por parte de los porteros (estos cambios se incluyen en el anexo y están señalados con un asterisco).

Para el bloque de toma de decisiones, al ser preguntados por la información empleada en la misma, el total de porteros hizo referencia al automatismo empleando expresiones como "es instinto" o "no sé, eso se sabe, te sale solo". Sin embargo, cuando se solicitó que desarrollaran en un dibujo un esquema de situación a modo de ejemplo, todos ellos señalaron con facilidad índices perceptivos que usaban en la toma de decisiones. El empleo de pre-índices perceptivos en la toma de decisiones ha sido ampliamente demostrado en la literatura en deporte en general y en fútbol en particular, incluyendo el estudio de los índices del tirador empleados por parte del portero (Murgia et al, 2014; Navia et al., 2011; Nöel y Van der Kamp, 2012). Por ello, se modifica este aspecto de la entrevista semi-estructurada para dar cabida a dichos aspectos.

En el bloque de manejo de situaciones competitivas, de nuevo una de las preguntas requirió sistemáticamente de especificaciones, puesto que al indagar en el afrontamiento del error los porteros hicieron referencia a la relatividad de los mismos. Cuando la entrevistadora solicitó aclaraciones acerca de los tipos de errores, de forma natural los porteros hicieron referencia a errores trascendentales y errores no trascendentales. La diferenciación entre errores trascendentales y no trascendentales así como sus consecuencias se han abordado desde la Psicología del Deporte, especialmente en sus faceta de diseño e implementación de la intervención dado el impacto que los primeros tienen sobre el global de la conducta deportiva (Bohórquez, 2017; Edwards, Hardy, Kingston, y Gould, 2002; Moreno, Conte, Silveira y Ruiz, 2014) y los segundos sobre el estado del aprendizaje motor (Martin, 2008). Por ello, se procedió a modificar las preguntas de la entrevista para incluir ambos tipos de errores y su influencia sobre el partido.

Este trabajo presenta algunas limitaciones. En primer lugar se trata de una primera aproximación con una muestra piloto reducida de cuatro porteros, aunque los estudios con la entrevista como método de recogida suelen tener muestras más limitadas que los estudios que utilizan las medidas autoinformadas. Por otra parte, quizá un número mayor de evaluadores en la fase de valoración de la prioridad de intervención habría aumentado el rango de observaciones y por tanto, habría aumentado la validez de los resultados.

En estudios futuros sería interesante confirmar la fiabilidad test-retest y la validez con una muestra mayor de porteros, en la línea de lo planteado por Ezpeleta et al (1997) e incluso ampliar la muestra con porteros de diferentes disciplinas con balón como balonmano, fútbol sala o waterpolo. 
Además, también sería interesante comprobar su estabilidad temporal en función del momento de la temporada en la cuál es administrada, tanto si es durante ciclos de entrenamiento, como antes o después de la competición.

\section{Aplicaciones prácticas}

Aunque es cierto que el fútbol ha recibido amplia atención desde la Psicología aplicada (Roffé, Del Vega, García-Más y Llinás, 2007; Roffé, 2016), este trabajo presenta dos aspectos que no han sido explorados: el portero de fútbol y la entrevista cualitativa por puesto. Los estudios acerca del portero de fútbol se han incrementado en los últimos años, aunque aún son necesario abordajes prácticos para esta posición específica en fútbol y otros deportes (García-Angulo y Ortega, 2015). Este trabajo aporta una nueva herramienta específica para la evaluación de las competencias psicológicas de los porteros de fútbol, atendiendo a la importancia de realizar evaluaciones concretas y adaptadas que abarquen el total de la conducta de los deportistas (Garcés de los Fayos, 2006; Martin, 2008) y ampliando el foco de atención desde los penaltis hasta la conducta global de éstos jugadores.

Partiendo de una evaluación concreta y exhaustiva es posible el desarrollo de mejores programas de intervención tal y como señalan Anguera (2002) y Tkachuck et al. (2003) entre otros.

\section{Referencias}

Angold, A. y Costello, J. E. (2000). The Child and Adolescent Psychiatric Assesment (CAPA), Journal of the American Academy of Child and Adolescent Psychiatry, 39, 39-48.

Anguera, M. T. (2002). Evaluación en psicología del deporte. En J. Dosil (Ed.), El psicólogo del deporte. Asesoramiento e intervención (pp. 51-68). Madrid, España: Síntesis.

Anguera, M. T., Camerino, O., Castañer, M. y Sánchez-Algarra. P. (2014). Mixed methods en la investigación de la actividad física y el deporte. Revista de Psicología del Deporte, 23, 123-130.

Ato, M., López, J.J. y Benavente, A. (2013). Un sistema de clasificación de los diseños de investigación en psicología. Anales de Psicología, 29, 1038-1059.

Bar-Eli, M., Azar, O. H., Ritov, I., Keidar-Levin, Y. y Schein, G. (2007). Action bias among elite soccer goalkeepers: The case of penalty kicks. Journal of Economic Psychology, 8, 606-621.

Bohórquez, M. R. (2016). Ser portero de fútbol (o ser uno en un equipo). Implicaciones para el trabajo psicológico. Boletín informativo de la FEPD, 24(dic.), 26-28.

Bohórquez, M. R. (2017). Errores deportivos, evaluación y manejo desde la psicología del Deporte. Taller impartido en el XV Congreso Andaluz y II Luso-Andaluz de Psicología de la Actividad Física y el Deporte, Granada, España.
Cantón, E. (2016). La especialidad profesional en psicología del deporte. Revista de Psicología Aplicada al Deporte y al Ejercicio Físico, 1, e2. https://doi.org/10.5093/rpadef2016a2

Capdevila, L. y Niñerola, J. (2006). Metodología de evaluación en psicología del deporte y de la actividad física: criterios y herramientas. En E. J. Garcés de los Fayos, A. Olmedilla y P. Jara (Eds.), Psicología y deporte. Murcia, España: Diego Marín.

Cohen, J. (1960). A coefficient of agreement for nominal scales. Educational and psychological measurement, 20, 37-46.

Dosil, J. (2004). Psicología de la actividad física y del deporte. Madrid, España: McGrawHill.

Edwards, T., Hardy, L., Kingston, K. y Gould, D. (2002). A qualitative analysis of catastrophic performances and the associated thoughts, feelings, and emotions. The Sport Psychologist, 16, 1-19.

Eklund, R. C. (2014). Introduction. En G. Tenenbaum y R. C. Eklund (Eds.), Encyclopedia of sport and exercise psychology (pp. xxiiixxvii). Los Angeles, CA, Estados Unidos: SAGE.

Ezpeleta, L., Osa, N. de la., Doménech, J. M., Navarro, J. B. y Losilla, J. M. (1997). Fiabilidad test-retest de la adaptación española de la Diagnostic Interview for Children and Adolescents DICA-R. Psicothema, 9, 529-539.

Furley, P., Dicks, M. y Memmert, D. (2012). Nonverbal behavior in soccer: the influence of dominant and submissive body language on the impression formation and expectancy of success of soccer players. Journal of Sport \& Exercise Psychology, 34(1), 61-82

Garcés de Los Fayos, E. (2006). Evaluación en psicología del deporte. En A. Olmedilla, E. J. Garcés de los Fayos y P. Jara. Psicología y Deporte (pp. 429-446). Murcia: Diego Marín Librero Editor.

García-Angulo, A. y Ortega, E. (2015). Análisis bibliométrico de la producción científica sobre el portero en fútbol. Revista Iberoamericana de Psicología del Ejercicio y el Deporte, 10(2), 205214.

García-Más, A. (2002). La psicología del fútbol. En J. Dosil (Ed.) El psicólogo del deporte. Asesoramiento e intervención (pp. 101131). Madrid, España: Síntesis.

González, M. D. (2010). Evaluación psicológica en el deporte: aspectos metodológicos y prácticos. Papeles del Psicólogo, 31, 250-258.

Jiménez, J. y Garcés de los Fayos, E. J. (1997). El papel profesional del psicólogo del deporte. En E. J. Garcés de los Fayos (Ed.), Manual de Psicología del Deporte: conceptos y aplicaciones (pp. 55-84). Murcia, España: Capítulo Tres.

Landis, J. y Koch, G. (1977). The measurement of observer agreement for categorical data. Biometrics, 33, 159-174.

Lidor, R., Ziv, G. y Gershon, T. (2012). Psychological Preparation of Goalkeepers for the 11-m Penalty Kick in Soccer - A Review. The Sport Psychologist, 26, 375-389.

Manley, A. J., Greenlees, I., Graydon, J., Thelwell, R., Filby, W. C. D. y Smith, M. J. (2008). Athletes' Perceived Use of Information Sources When Forming Initial Impressions and Expectancies of a Coach: An Explorative Study. The Sport Psychologist, 22, 73-89.

Martin, G. L. (2008). Psicología del deporte. Guía práctica del análisis conductual. Madrid, España: Pearson, Prentice-Hall.

Meredith, S. J., Dicks, M., Noel, B. y Wagstaff, C. R. (2017). 
A review of behavioural measures and research methodology in sport and exercise psychology. International Review of Sport and Exercise Psychology, 1-22. http://dx.doi.org/10.1080/1750984X.2017.1286513

Moreno, J. A., Conte, L., Silveira, Y. y Ruiz, L. M. (2014). Miedo a fallar en el deporte. Elche, España: Universidad Miguel Hernández de Elche.

Murgia, M., Sors, F., Muroni, A. F., Santoro, I., Prpic, V., Galmonte, A. y Agostini, T. (2014). Using perceptual home-training to improve anticipation skills of soccer goalkeepers. Psychology of Sport and Exercise, 15, 642-648.

Navia, J. A., Ruiz, L. M., Graupera, J. L. y Van der Kamp, J. (2011). La mirada de los porteros de fútbol sala ante diferentes tipos de respuesta motriz. International Journal of Sport Science, IX(33), 216-229.

Nöel. B. y Van der Kamp, J. (2012). Gaze behaviour during the soccer penalty kick: An investigation of the effects of strategy and anxiety. International Journal of Sport Psychology, 41, 1-20.

Perpiñá, C. (2014). Manual de la entrevista psicológica. Madrid, España: Pirámide.

Ryu, D., Kim, S., Abernethy, B. y Mann, D. L. (2013). Guiding Attention Aids the Acquisition of Anticipatory Skill in Novice Soc- cer Goalkeepers. Research Quarterly for Exercise \& Sport, 84(2), 252-262.

Roffé, M. (2016). La preparación psicológica de la Selección Nacional Absoluta de Colombia para el Mundial de Fútbol Brasil 2014. Revista de Psicología Aplicada al Deporte y al Ejercicio Físico, 7, e3. https://doi.org/10.5093/rpadef2016a3

Roffé, M., De la Vega, R., García-Mas, A. y Llinás, J. (2007). Las crisis durante el juego: el gol psicológico en el fútbol. Revista de Psicología del Deporte, 16, 227-240.

Sánchez, X. y Torregrosa, M. (2005). El papel de los factores psicológicos en la escalada deportiva: un análisis cualitativo. Revista de Psicología del Deporte, 14, 177-194.

Tkachuck, G., Leslie-Toogood, A. y Martin, G. L. (2003). Behavioral Assessment in Sport Psychology. The Sport Psychologist, 17, 104-117.

Torregrosa, M., Sánchez X. y Cruz, J. (2004). El papel del psicólogo del deporte en el asesoramiento académico - vocacional del deportista de elite. Revista de Psicología del Deporte, 13(2), 215-228.

Williams, J. M. (1991). Integración y puesta en práctica de un programa de entrenamiento en destrezas psicológicas. En J. M. Williams (Ed), Psicología aplicada al deporte (pp.-507-539). Madrid, España: Biblioteca Nueva. 


\section{Anexo}

Entrevista para la Evaluación Psicológica de Porteros de Fútbol.

Datos personales y de contacto.

Deportista

Fecha

Fecha de nacimiento

Estado civil

Nivel académico

Teléfono de contacto

Email de contacto

\section{Datos sociodeportivos.}

Club actual

Edad con que empezó a jugar al fútbol

Competiciones en las que participa

Años en la categoría actual
Clubes anteriores

Edad con que empezó a jugar como portero

Titular

Suplente

\section{Tiempo máximo sometido a suplencia}

Para entrenar las tareas conjuntas

Para que el equipo entrene la finalización

$N^{0}$ y duración de entrenamientos específicos a la semana:

\section{Motivo de consulta y/o información de derivación.}

\section{Si viene por decisión propia}

¿Qué te ha hecho pensar en trabajo psicológico?

¿Qué opina tu entrenador (u otro) de que hagas trabajo psicológico?

\section{Si acude por derivación}

¿Por qué crees que tu portero se beneficiaría de un trabajo psicológico? 
Motivación: causas del inicio y mantenimiento en la actividad deportiva, factores influyentes en los procesos motivacionales y manejo de las variaciones.

¿Qué es lo que más te gusta de tu deporte?, ¿y lo que menos?

$\rightarrow$ ¿Por qué?, ¿siempre ha sido así?

¿Qué es lo que te gusta de ser portero?, ¿y lo que menos?

$\rightarrow$ ¿Por qué? ¿siempre ha sido así?

¿Cuáles son tus objetivos deportivos?

$\rightarrow$ ¿Dónde te gustaría llegar a nivel deportivo?, ¿y un plazo de aproximadamente un año? ¿y en los próximos meses?

¿Siempre tienes energía para trabajar por lograr esos objetivos?, ¿qué cosas te dan energía?, ¿hay cosas que te la quitan?

$\rightarrow$ ¿Has probado algo que te ayude a estar motivado en el entrenamiento/partido?

\section{Autoconcepto y autoestima: fortalezas y debilidades como portero, situación respecto de la situación de referencia.}

¿Cuáles son tus puntos fuertes como portero?, ¿y los débiles?

$\rightarrow$ *Físico (flexibilidad, coordinación, potencia...).

$\rightarrow$ *Técnica (aquellas habilidades de manejo del balón como pasar, blocar...).

$\rightarrow$ *Táctica (gestionar a tus compañeros y a los atacantes).

$\longrightarrow$ *Estrategia (manejar el juego en función del tiempo, el espacio, los otros...).

$\rightarrow$ *Psicológico (conocerte y gestionarte a ti mismo, como la concentración, la capacidad de soportar el estrés...).

¿Quién es para ti una referencia como portero?, ¿en qué crees que os parecéis?, ¿en qué os diferenciáis?

¿Qué nivel crees que tienes respecto a los porteros de tu categoría?, ¿por qué?

¿Cuándo te evalúas a ti mismo como portero, qué resultado obtienes?

$\rightarrow$ ¿Cómo te sientes?, ¿qué se te pasa por la cabeza?

Transferencia de las habilidades deportivas y psicológicas del entrenamiento a la competición: estado del aprendizaje y control motor de las acciones específicas.

¿Compites como entrenas?

$\rightarrow$ ¿En qué se diferencian?

¿Te salen en los partidos las cosas que has estado entrenando?

$\rightarrow$ ¿Qué es exactamente lo que falla?

$\rightarrow$ ¿Tienes que pensarlas o las ejecutas de forma automática?

$\rightarrow$ ¿Eres capaz de hacer lo que deseas en el momento que deseas o te aceleras o ralentizas? 
Atribuciones causales: atribuciones ante éxitos y fracasos, consecuencias conductuales cognitivas y afectivas, errores de atribución. Identificación de componentes de flow asociados a la mejor competición.

¿Cuál ha sido tu mejor partido en los últimos 12 meses?, ¿cuáles crees que fueron las causas?, ¿cómo te sentías?

$\rightarrow$ ¿Creaste esa situación o simplemente la encontraste?

Durante ese partido...

¿Sentías que podías perfectamente hacer lo que requería el partido? Sí No

¿Tenías claro tu objetivo en ese partido? Sí No

¿Veías de forma evidente las consecuencias de tus acciones? Sí No

¿Tomabas decisiones sin tener que pensarlas o dudar? Sí No

¿Te sentías absorbido en el partido? Sí No

¿El tiempo parecía no contar, como si no importara? Sí No

¿Sentías que tenías bajo control ese partido? Sí No

¿Disfrutaste al máximo de ese partido? Sí No

¿Y tú peor partido en ese tiempo?, ¿cuáles crees que fueron las causas?, ¿cómo te sentías?

$\rightarrow$ ¿Trataste de hacer algo para cambiar la situación?

Arousal: identificación de la zona óptima de funcionamiento individual, aspectos que sacan el portero de la zona, estrategias de recuperación del estado de óptimo funcionamiento.

Algunos porteros son capaces de identificar cuándo están listos para hacer un buen entrenamiento o partido usando las sensaciones físicas y mentales que tienen. ¿Tú podrías identificar en ti mismo ese estado?

$\rightarrow$ ¿Qué claves usas?

Si a esa sensación la llamáramos estar en zona. ¿Cómo sabes cuándo estás fuera de zona?

$\rightarrow$ ¿Y por encima/debajo de tu zona? (en contraposición al aspecto señalado anteriormente).

¿Hay cosas o situaciones que te sacan de tu zona?, ¿y cosas o situaciones que te meten en zona?

$\rightarrow$ ¿Has tratado alguna vez de manejarlas?, ¿cómo?, ¿funcionó?

Toma de decisiones: procedimientos y estilos, influencia de otros, influencia de las emociones.

¿Te cuesta trabajo tomar decisiones en los entrenamientos?, ¿y en los partidos?

$\rightarrow$ ¿Crees que, en general, aciertas cuando tomas decisiones?

$\rightarrow$ ¿Es diferente si es una situación que has entrenado o una que no has trabajado?

Hasta que tomas la decisión, ¿te sientes presionado?

$\rightarrow$ ¿Tener poco tiempo para decidir aumenta tu estrés?

$\rightarrow$ ¿Qué un compañero o el entrenador te dé indicaciones mientras piensas aumenta tu agobio?

$\rightarrow$ Tener que decidir sin tener certeza del resultado te afecta?

¿Qué información sueles usar para tomar decisiones?, ¿en qué te basas para saber qué hacer?

* $\rightarrow$ ¿Tienes en cuenta tu posición respecto de la portería para tomar decisiones?, ¿y la situación de los compañeros?, ¿y la de los atacantes?

* $\rightarrow$ ¿Hay aspectos del cuerpo del lanzador que te faciliten tomar decisiones?

$\rightarrow$ ¿Hay otros aspectos que sea necesario tener en cuenta?, ¿el terreno?, ¿el vuelo del balón?... 
¿Tomas decisiones igual cuando tienes emociones negativas que cuando son positivas?

$\rightarrow$ *Tras un error trascendental, ¿tomas decisiones de la misma manera?

$\rightarrow$ En un resultado adverso para el equipo, ¿tomas decisiones de la misma manera?

$\rightarrow$ Cuando cambia tu nivel de confianza, ¿tomas decisiones de la misma manera?

$\rightarrow$ Ante rivales que conocen o suelen explotar tus puntos débiles, ¿tomas decisiones igual que ante cualquier otro jugador?

$\rightarrow$ ¿Tomas decisiones igual en función de los compañeros que estén en defensa ese día?

Ansiedad precompetitiva: presencia/ausencia, sintomatología, impacto en los componentes de entrenamiento invisible, impacto en la precompetición, impacto en la competición, impacto en la postcompetición y estrategias de control.

¿Te notas ansioso antes de los partidos?, ¿qué te ocurre?

$\rightarrow$ ¿Sientes más sudor en las manos del habitual?, ¿ganas de ir al baño?, ¿el estómago revuelto o cerrado?...

$\rightarrow$ ¿Sientes que la cabeza te va rápido o se bloquea?, ¿piensas en lo que puede ir mal?

$\rightarrow$ ¿Qué haces cuando eso te pasa?, ¿funciona?

¿Y el día/mañana antes del partido te notas intranquilo o ansioso?, ¿qué te ocurre?

$\rightarrow$ ¿Te cuesta quedarte dormido?, ¿te despiertas en mitad de la noche?, ¿te despiertas antes de que suene el despertador, ¿te levantas con sensación no haber descansado?

$\rightarrow$ ¿Comes normalmente?, ¿mantienes el mimo apetito?

$\rightarrow$ ¿Estás más irritable?

$\rightarrow$ ¿Qué haces cuando eso te pasa?, ¿funciona?

(En caso de haber respondido afirmativa a la/s anterior/es) ¿Esto influye en cómo calientas antes del partido? ¿y en tu partido?

$\rightarrow$ ¿Cómo te afecta?

¿Qué haces normalmente después de los partidos?

$\rightarrow$ ¿Cambia en función del resultado del equipo?

$\rightarrow$ ¿Cambia en función de los goles que has encajado o los errores que puedas haber cometido?

\section{Manejo de las situaciones competitivas: distracciones, estrategias de reenfoque, gestión del error, gestión del éxito, nivel de confianza percibido.}

¿Te distraes durante los partidos?, ¿qué cosas te distraen?

$\rightarrow$ iTus pensamientos te distraen alguna vez?

¿Haces alguna cosa para conseguir concentrarte?, ¿qué?

* ¿Te afecta cometer errores trascendentales en la competición?, ¿de qué forma?

* ¿Y errores no trascendentales?, ¿de qué forma?

¿Te afectan los aciertos?, ¿de qué forma?

¿Haces algo para manejar esas situaciones en las que los errores 0 aciertos te afectan?, ¿funciona?

¿Qué nivel de confianza tienes normalmente durante la competición?, ¿de qué depende esa confianza?

$\rightarrow$ ¿Se mantiene durante todo el partido?

$\rightarrow$ Si la valoráramos de 0 a 10, ¿cuál sería el máximo?, ¿y el mínimo?

¿Haces algo para manejar esa confianza?, ¿funciona? 
Cuestiones relacionadas con agentes deportivos influyentes: compañeros (incluyendo otro/s porteros), entrenador de porteros, primer entrenador, preparador físico, fisioterapeuta, otros técnicos, familia y otros pertenecientes a la red de apoyo social.

\section{Relación con profesionales a su servicio}

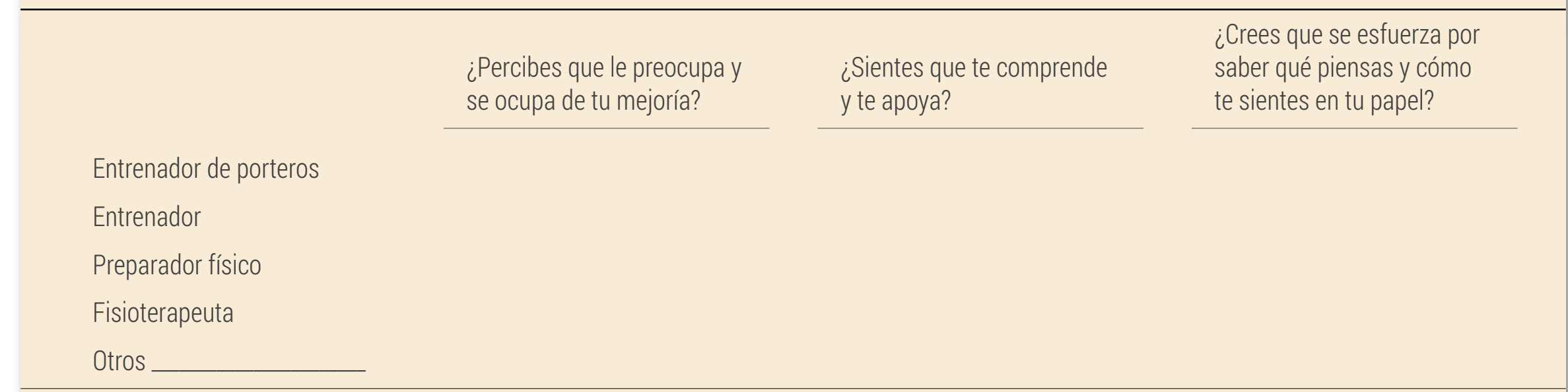

\section{Relación con compañeros}

¿Qué relación tienes con tus compañero/s porteros?

$\rightarrow$ ¿Sientes que sois un apoyo entre vosotros?

¿Y con el resto de compañeros?, ¿hay alguien en quien te apoyes especialmente?

\section{Relaciones familiares y de pareja}

Háblame de tu familia, ¿quién la compone? (Dibujar el genograma).

¿Es tu familia un apoyo en tu carrera deportiva?, ¿en qué sentido?

$\rightarrow$ ¿Se involucran en tu carrera?

$\rightarrow$ ¿Quién/es son tu apoyo?

¿Tienes pareja?, ¿es un apoyo en tu carrera deportiva?, ¿en qué sentido?

$\rightarrow$ ¿Se involucra en tu carrera?

\section{Otras relaciones importantes: amigos, representante...}

Percepción de necesidades psicológicas a entrenar.

¿Hay alguna cosa que creas que es importante y yo no te haya preguntado?

¿Qué piensas que deberías entrenar a nivel psicológico?

Observaciones del psicólogo tras la evaluación inicial. 